\title{
TG-MS analysis of the thermal decomposition of pig bone for forensic applications
}

\author{
A. Onishi ${ }^{1}$, P.S. Thomas ${ }^{1 *}$, B.H. Stuart ${ }^{2}$, J.P. Guerbois ${ }^{1}$ and S.L. Forbes ${ }^{3}$ \\ ${ }^{1}$ Department of Chemistry, Materials and Forensic Sciences, University of Technology, \\ Sydney, PO Box 123, Broadway NSW 2007, Australia \\ ${ }^{2}$ Centre for Forensic Science, University of Technology, Sydney, PO Box 123, Broadway \\ NSW 2007, Australia \\ ${ }^{3}$ University of Ontario Institute of Technology, Faculty of Science, 2000 Simcoe St North, \\ Oshawa ON L1H 7K4, Canada
}

* Corresponding author

\begin{abstract}
In order to investigate the potential of thermal analysis for the determination of post-mortem age, rib bone specimens were collected from the remains of a number of slaughtered pigs that were allowed to decompose in the Australian bush in a controlled site under a range of conditions for time periods ranging from 1 to 5 years. The bone specimens were cut in crosssection with the compact bone collected for analysis. TG-MS curves were collected by heating bone samples to $1100^{\circ} \mathrm{C}$ in an argon atmosphere. The TG-MS data showed significant differences for the pig bone specimens derived from the different environments and showed trends in peak size correlating with age. The reported data suggest that TG-MS has significant potential for the identification of origin as well as the ageing of skeletal remains in a forensic context.
\end{abstract}

Keywords: bone; forensic; mass spectrometry; thermogravimetric analysis.

\section{Introduction}

A problem for forensic examiners is the ageing and characterisation of bone fragments or decomposed skeletal remains. The difficulties lie in the complex nature of bone and in its interaction with the environment. Due to the sensitivity of thermal methods to morphological states, thermogravimetric analysis (TG) has been selected as a technique which may overcome these problems.

TGA has been utilised for the characterisation of bones in a number of studies [1-7]. TG has also been used in combination with mass spectrometry (MS) to obtain further 
information regarding the composition of bones [4-6]. The TG data of bones shows mass losses at particular temperatures that may be correlated with the organic and inorganic phases present in bone. The first step occurs from $50-260^{\circ} \mathrm{C}$ and is associated with water loss. A second step up to $600^{\circ} \mathrm{C}$ represents the combustion of the organic components to produce carbon dioxide, water and organic fragments. A third step in the range $650-850^{\circ} \mathrm{C}$ is observed and is associated with the release of carbon dioxide from the carbonated hydroxyapatite if the decomposition is carried out in an air atmosphere, but is predominantly associated with the further decomposition of the products of pyrolysis produced in the second step if the decomposition is carried out in an inert atmosphere [6].

In the current study, TG-MS has been used to characterise bone specimens obtained from pigs of different post-mortem age and subjected to different burial environments. Selected fragments have been monitored in order to find a correlation with post-mortem age.

\section{Experimental}

Samples of pig rib bone were provided by the Centre for Forensic Science at the University of Western Australia from the remains of an ongoing research project on the environmental effects of tissue decomposition. Female pigs, $45 \mathrm{~kg}$ in weight, were slaughtered and allowed to decompose in the Western Australian bush in a controlled site under a range of conditions which included: burial in a clothed and unclothed state; deposition on the soil surface, also in a clothed and unclothed state; and stored, clothed, in a car purged with carbon monoxide to imitate the decomposition of the pig under the conditions of 'car gas suicide'. The pig carcases were kept in these conditions for a range of time periods from 1 to 5 years. Table 1 summarises the details of the specimens. Rib bone specimens were collected from the remains and cut in cross-section with the compact bone collected for analysis. The compact bone specimens were cleaned only by scaping of the surface with a scalpel to remove fatty bone marrow from the interior and any residues from the exterior of the specimens.

The bone specimens were examined by TG using a Setaram Setsys 16/18 thermobalance coupled with a Balzers ThermoStar mass spectrometer for evolved gas analysis (EGA). Experiments were carried out by placing approximately $15-20 \mathrm{mg}$ of the bone sample into a platinum crucible and heating at a rate of $10^{\circ} \mathrm{C} \mathrm{min}^{-1}$ from ambient temperature to $1100^{\circ} \mathrm{C}$ under flowing $\left(20 \mathrm{~mL} \mathrm{~min}^{-1}\right)$ high purity argon gas. Temperature calibration was carried out using indium, tin, aluminium, gold and silver. Baseline curves 
measured under the same experimental conditions were acquired to account for buoyancy effects on the balance. The mass to charge ratios selected for analysis were 18, 28, 30, 32, 44, $54,64,67,70,72,78,81$ and 91 amu. A one second acquisition time for each mass unit was set, thus requiring $0.13 \mathrm{~min}\left(\right.$ or $1.3^{\circ} \mathrm{C}$ ) for each cycle.

\section{Results and Discussion}

The TG and differential TG (DTG) curves for the specimen SC1 are shown in Figure 1. These curves show the three distinct regions of decomposition; Region 1 from room temperature to $220^{\circ} \mathrm{C}$, Region 2 from 220 to $570^{\circ} \mathrm{C}$ and Region 3 from 570 to $1050^{\circ} \mathrm{C}$. Each of these regions is characteristic of a mass loss step, the total mass losses of which are listed in Table 2. The DTG curve is plotted with a selected range of MS mass to charge ratio (in amu) curves in Figure 2 for the specimen SC1, aiding the characterisation of each mass loss step.

Region 1 is associated with the evolution of water (18 amu) with a peak in the DTG curve observed at $120^{\circ} \mathrm{C}$. The peak in the 18 amu curve is at a higher temperature $\left(190^{\circ} \mathrm{C}\right)$. The higher observed temperature may be accounted for by the onset of decomposition processes which evolve water as well as organic species, resulting in a peak shift to higher temperature.

Region 2 is characterised by a two step decomposition process of the organic material identified with peaks in the MS curves which correspond to organic fragments. The two peaks observed in the DTG are centred on 348 and $451{ }^{\circ} \mathrm{C}$ in the decomposition of SC1. Inspection of the MS curves in Figure 2 indicates that there are two types of MS curves attributable to the decomposition of the organic fragments. Of these MS curves, the curves of the 72 and 78 amu mass fragments have been selected for comparison of the range of pig bone specimens listed in Table 1 (Figures 3 and 4). In addition to the organic fragments, $\mathrm{CO}_{2}$ (44 amu curve) is also observed to be evolved in Region 2. $\mathrm{CO}_{2}$ may be evolved as either a product of the decomposition of the inorganic phase (the carbonated hydroxyapatite) or as a product of pyrolysis. A third possibility is the oxidation of the organic matter by atmospheric $\mathrm{O}_{2}$. Although the experiments were carried out in an argon atmosphere using high purity argon, the argon purge gas still contains a small amount of oxygen $(<10 \mathrm{ppm})$. It is this oxygen that is likely to be responsible for the $\mathrm{CO}_{2}$ produced through oxidation of the organic matter. This is supported by the 32 amu curve, attributed to $\mathrm{O}_{2}$, which is observed to have negative peaks that correspond to the positive peaks in the $\mathrm{CO}_{2}$ curve. This is confirmed by 
the presence of a sharp negative peak in the $\mathrm{O}_{2}$ curve at $483^{\circ} \mathrm{C}$ which corresponds well with a positive peak at the same temperature in the $\mathrm{CO}_{2} \mathrm{MS}$ curve.

The thermal decomposition of the specimen SC1 is observed throughout Region 3 as the DTG curve is not observed to reach zero even at temperatures above $1000^{\circ} \mathrm{C}$. In this region a broad decomposition step is observed from 680 to $1060^{\circ} \mathrm{C}$ with the peak centred on $875^{\circ} \mathrm{C}$. Although there is likely to be some decomposition of the mineral phase in this region, the mass loss appears to be predominantly associated with the evolution of organic products as the $\mathrm{O}_{2}$ MS curve once again shows a negative peak in line with the positive peak observed in the $\mathrm{CO}_{2} \mathrm{MS}$ curve.

The aim of this work was to apply TG-MS to the characterisation of the series of bone samples listed in Table 1 and to identify the potential of TG-MS as an appropriate tool for the determination of the post mortem age of the bone specimens based on the assumption that as the bone specimens age in situ, chemical change would be observed in the thermal decomposition products in the MS. Additionally, as thermal methods are sensitive to morphology due to the dynamic nature of the data acquisition, differences would be expected to be observed due to a change in the diffusion path length of volatiles produced in the decomposition process, resulting in shifts in mass loss peaks. For this purpose the comparative data are plotted in Figures 3 and 4 for the 72 and 78 amu MS curves, as representative curves of the thermal decomposition of the organic phase, with the DTG curves plotted in Figure 5.

The DTG curves show some significant variance between specimens which is particularly notable in Regions 1 and 2. Five individual decomposition steps can be identified across Regions 1 and 2 at $100,145,265,350,430^{\circ} \mathrm{C}$ with the first two steps corresponding to Region 1 (water loss) and the latter three to Region 2 (organic phase pyrolysis). These peaks appear with differing intensities in each of the DTG curves. The specimen GC1.5 is significantly different showing prominent evidence of all five observable peaks. These observed differences might be expected as the decomposition environment of GC1.5 is significantly different; the carcass was sealed in a car which was filled with carbon monoxide and this carcass had no soil contact. The DTG curves of the surface deposited and the buried specimens are more similar, but also have some significant differences in their decomposition character; a peak is observed at $255^{\circ} \mathrm{C}$ in the 78 amu curves for the SC4 and SC5 specimens, but is absent in the buried specimens' curves (Figure 4). Although this is a small sample of test specimens, the relatively simple differentiation between the origins demonstrates the sensitivity of TG-MS to origin of the specimens. 
Ageing of the specimens by TG-MS also appears promising and certain trends are apparent. The shoulder at approximately $430^{\circ} \mathrm{C}$ in the DTG curves diminishes in size with increasing age for the SC series. The corresponding peak in the 72 and 78 amu MS curves is also diminished in size for this series. The pair of peaks in the 72 and 78 amu MS curves corresponding to the 350 and $430^{\circ} \mathrm{C}$ DTG peaks is also indicative of age and appears to be independent of the environment. These are qualitative assessments based on a visual inspection of the shapes of these peaks and, hence, a more rigorous, quantitative study needs to be undertaken with a wider range of specimens for the confirmation of these trends. However, the observation that TG-MS appears to be sensitive to both age and environment should encourage further investigation into the application of this technique to the ageing and identification of the origin of skeletal remains in a forensic context.

\section{Conclusions}

A preliminary study has been carried out to identify the potential of TG-MS for the determination of origin and age of skeletal remains. The investigation has identified significant differences between data based on origin and has indicated that ageing trends do appear in the data. TG-MS has, therefore, shown the potential and deserves further investigation.

\section{Acknowledgements}

The authors would like to thank the Centre for Forensic Science, University of Western Australia, for the provision of bone samples.

\section{References}

1. G.E. Fantner, H. Bikedal, J.H. Kindt, T. Hassenkam, J.C. Weaver, J.A. Cutroni, B.L. Bosma, L Bawazer, M.M. Finch, G.A.G. Cidade, D.E. Morse, G.D. Stucky and P.K. Hansma, Bone, 35 (2004) 1013.

2. Y. Okamoto, S. Hidaka, Y. Yamada, K. Ouchi, K. Miyazaki and S.Y. Liu, J. Biomed. Mater. Res., 41 (1998) 221.

3. M. Utech, D. Vuono, P. De Luca and A. Nastro, J. Therm. Anal. Cal., 80 (2005) 435.

4. L.D. Mkukuma, J.M.S. Skakle, I.R. Gibson, C.T. Imrie, R.M. Aspden and D.W.L Hukins, Calc. Tiss. Inter., 75 (2004) 321.

5. F. Peters, K. Schwarz and M. Epple, Thermochim. Acta, 361 (2000) 131. 
6. A. Onishi, P.S. Thomas, B.H. Stuart, J.P. Guerbois and S.L. Forbes, J. Therm. Anal. Cal., 88 (2007) 405.

7. C.Y. Ooi, M. Hamdi and S. Ramesh, Ceramics Int., in press. 


\section{List of figures}

Figure 1. TG and DTG curves for SC1 bone.

Figure 2. Temperature dependence of ion current for various fragments of SC1 bone.

Figure 3. Temperature dependence of the 72 amu fragment.

Figure 4. Temperature dependence of the 78 amu fragment.

Figure 5. DTG curves for all bone specimens. 
Table 1. Bone sample details.

\begin{tabular}{clc}
\hline Sample & Burial Conditions & Burial Time (years) \\
\hline SC1 & clothed, surface deposit & 1 \\
SC4 & clothed, surface deposit & 4 \\
SC5 & clothed, surface deposit & 5 \\
BU3 & unclothed, buried & 3 \\
BU4 & unclothed, buried & 4 \\
BC5 & clothed, buried & 5 \\
GC1.5 & clothed, simulated car suicide by & 1.5 \\
& carbon monoxide gas & \\
\hline
\end{tabular}


Table 2. Total mass loss up to 220,570 and $1050^{\circ} \mathrm{C}$.

\begin{tabular}{|c|c|c|c|}
\hline Sample & $\begin{array}{c}\text { Total mass loss up to } \\
220^{\circ} \mathrm{C}(\%)\end{array}$ & $\begin{array}{c}\text { Total mass loss up to } \\
570^{\circ} \mathrm{C}(\%)\end{array}$ & $\begin{array}{c}\text { Final mass loss at } \\
1050^{\circ} \mathrm{C}(\%)\end{array}$ \\
\hline SC1 & 11.5 & 34.8 & 43.3 \\
\hline SC4 & 8.7 & 33.4 & 42.1 \\
\hline SC5 & 8.7 & 29.8 & 38.0 \\
\hline BU3 & 11.5 & 32.3 & 41.5 \\
\hline BU4 & 9.4 & 29.8 & 38.4 \\
\hline BC5 & 10.6 & 29.9 & 38.6 \\
\hline GC1.5 & 8.5 & 37.9 & 45.7 \\
\hline
\end{tabular}

